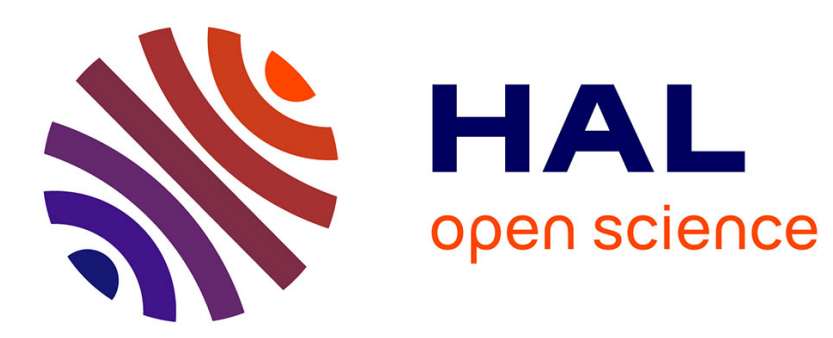

\title{
A methodology for Raman structural quantification imaging and its application to iron indoor atmospheric corrosion products
}

Judith Monnier, Ludovic Bellot-Gurlet, Denis Baron, Delphine D. Neff, Ivan

Guillot, Philippe Dillmann

\section{To cite this version:}

Judith Monnier, Ludovic Bellot-Gurlet, Denis Baron, Delphine D. Neff, Ivan Guillot, et al.. A methodology for Raman structural quantification imaging and its application to iron indoor atmospheric corrosion products. Journal of Raman Spectroscopy, 2011, 42 (4), pp.773 - 781. 10.1002/jrs.2765 . hal-01665918

\section{HAL Id: hal-01665918 https://hal.science/hal-01665918}

Submitted on 26 Aug 2018

HAL is a multi-disciplinary open access archive for the deposit and dissemination of scientific research documents, whether they are published or not. The documents may come from teaching and research institutions in France or abroad, or from public or private research centers.
L'archive ouverte pluridisciplinaire HAL, est destinée au dépôt et à la diffusion de documents scientifiques de niveau recherche, publiés ou non, émanant des établissements d'enseignement et de recherche français ou étrangers, des laboratoires publics ou privés. 


\title{
A methodology for Raman structural quantification imaging and its application to iron indoor atmospheric corrosion products
}

\author{
Judith Monnier, ${ }^{\mathrm{a}, \mathrm{c}}$ Ludovic Bellot-Gurlet, ${ }^{\mathrm{b}}$ Denis Baron, ${ }^{\mathrm{b}}$ \\ Delphine Neff, ${ }^{,}$Ivan Guillot, ${ }^{a}$ and Philippe Dillmann, ${ }^{c}$
}

\begin{abstract}
Raman structural imaging can bring original information to answer new questions raised with the recent studies on iron and low alloy steel corrosion. Up to now, this technique has allowed the extraction of the qualitative distribution of the compounds constituting the corrosion product layers. We propose here a methodology to extract quantitative parameters from Raman hyperspectral maps, executed by a homedeveloped software: LADIR-CAT. Specifically developed for iron corrosion quantitative component imaging, the approach and program operation are described. The LADIR-CAT is applied on ancient iron corroded samples originating from the Amiens cathedral (France) to establish the description and the composition of the corrosion system through quantitative compounds imaging. Moreover, the global phase quantities proposed supply data for calculating the so-called "protectivity ratio".
\end{abstract}

Keywords: Raman micro-spectroscopy; structural imaging; compound content quantification; archaeological artefacts; iron atmospheric corrosion

\section{Introduction}

Raman micro-spectroscopy is a technique well-suited for the characterisation of various oxides and (oxy)hydroxides involved in iron corrosion ${ }^{[1]}$. Moreover, this technique enables to describe the compounds distribution, using the acquisition of hyperspectral maps $^{[2]}$. This ability can be used to identify and localise the phases considered as active in corrosion processes, such as akaganeite in rich chlorine medium ${ }^{[3,4]}$ or lepidocrocite in atmospheric systems ${ }^{[5]}$, and to define their action in the corrosion processes. These active phases are often mixed at a micrometric scale, with stable ones. As a consequence, in order to discuss the stability of the corrosion system, one should gain some knowledge on the relative quantities of these active and stable phases in the altered system. Because Raman micro-spectroscopy is easily implemented, and demonstrates its great ability to identify iron based compounds at a micrometric scale, we propose in this paper a methodology of solid state compound quantification based on this technique.

In the continuity of a previous study ${ }^{[2]}$, we will focus on iron long term atmospheric corrosion. Indeed, iron is widely present in historical buildings and the management of an efficient preventive conservation requires a reliable degradation diagnosis and an operative prediction of the corrosion mechanisms. Moreover, in France, vitrified nuclear waste could be embedded in low carbon steel over-container exposed to indoor atmospheric conditions for several hundred of years. Therefore to predict the over-containers lifetime, the degradation over multisecular periods has to be modelled.
Current modelling of the atmospheric corrosion mechanisms deals with the wet-dry cycle proposed by Evans and Taylor ${ }^{[6]}$ and demonstrated by Stratmann et al. ${ }^{[7]}$. During this cycle some phases, identified as the lepidocrocite $(\gamma-\mathrm{FeOOH})$ by these authors, may play an active role in corrosion mechanisms. In contrast some of the phases such as the goethite $(\alpha-\mathrm{FeOOH})$, present in the corrosion product layers, are considered as electrochemically stable. As a consequence, the fine characterisation of the different phases in the corrosion layers and their quantification are crucial steps for a better understanding of the corrosion layer reactivity and iron long term corrosion mechanisms. In particular, the ratio of the less active versus the more active phases could provide reliable data concerning the corrosion scale reactivity. This ratio, named "protective ability ratio" by Yamashita et al. ${ }^{[8]}$, was first assimilated to the goethite to lepidocrocite ratio, using quantities extracted from X-ray diffraction (XRD) measurements on rust powders. Other authors ${ }^{[9-11]}$ worked to improve its definition, taking into account other phases such as akaganeite $(\beta-\mathrm{FeOOH})$ or magnetite $\left(\mathrm{Fe}_{3} \mathrm{O}_{4}\right)$. These studies, based on the

\footnotetext{
a Université Paris-Est, Institut de Chimie et des Matériaux Paris-Est (ICMPE) UMR 7182 CNRS and Université ParisEst Créteil, 2 rue Henri Dunant, 94320 Thiais, France, monnier@icmpe.cnrs.fr, guillot@icmpe.cnrs.fr

b Laboratoire de Dynamique, Interactions et Réactivité (LADIR) UMR 7075, CNRS and UPMC - Université Pierre et Marie-Curie Paris 6, 2 rue Henri Dunant, 94320 Thiais, France, bellot-gurlet@glvt-cnrs.fr, baron@glvt-cnrs.fr

c Laboratoire Archéomatériaux et Prévision de l'Altération (LAPA), SIS2M UMR 3299 CEA-CNRS, and IRAMAT UMR5060 CNRS, CEA Saclay, 91191 Gif sur Yvette cedex, France,delphine.neff@cea.fr, philippe.dillmann@cea.fr
} 
accurate determination of the quantities of the phases present in the corrosion layers by XRD measurements or on Mössbauer spectroscopy, had nevertheless a limitation. The quantifications were based on the analysis of macroscopic samples of corrosion products. As conductive or electrochemically reactive phases are present inside the corrosion layers, the issues of their distribution and of their possible connection to the metallic substrate are crucial. For this purpose, the analysis at a micrometric scale of the various quantities of phases is of paramount interest. Moreover XRD or Mössbauer are not well-suited to fit complex mixtures at a micrometric scale, especially since XRD is not sensitive enough to low crystallised compounds. Because Raman micro-spectroscopy can identify, at a micrometric scale, all the phases involved even the low crystallised ones, we have developed a specific program to get semi-quantitative information from Raman hyperspectral maps ${ }^{[12]}$. Its aim is to evaluate the respective amounts of reactive phases compared to the less reactive ones in the corrosion scales.

We first describe the approach followed for solid state compound quantification, along with the program developed for hyperspectral maps' treatment. This approach will be then be applied to ancient samples coming from the Amiens' cathedral, in the North of France. On this historic site, nine samples submitted to indoor atmospheric corrosion over five centuries have been analysed leading to the identification of four phase families: a matrix of goethite considered as non reactive, and lepidocrocite, akaganeite and low crystallised phases considered as reactive ${ }^{[13,14]}$.

\section{Experimental}

Possible approaches for quantification

A first basic approach for compound quantification from Raman spectral maps should be the identification of the corresponding product for each analysed point, and their counting over the map. This can be efficient when the analysed volume contains only one component. But for the samples considered in this study, even with a beam size of about one micrometers diameter, most of the spectra correspond to a mixture of phases, as illustrated in Fig. 1a. The use of direct spectrum recognition and counting to obtain phase proportion is therefore impossible.

Moreover the difficulty is enhanced by the fact that iron corrosion products spectra exhibit bands at similar positions (see Fig. 2 for considered phase spectra), rending the use of individual bands improper for quantification. Quantification therefore requires multivariate analyses, where spectra are treated as combination of phase responses.

Making the assumption that the spectrum resulting from a mixture of phases is the sum of each phase spectrum, the experimental spectrum can be calculated from a linear combination of single phase spectra (Fig. 1b). From this consideration, the Raman scattering efficiency in the experimental conditions has to be taken into account for each phase. For that two approaches could be used.

The first is based on the recording of "calibration curves" representative of the mixtures encountered in the corrosion layers. For solids it can be achieved through a set of compounds mixed at various proportions. Such an
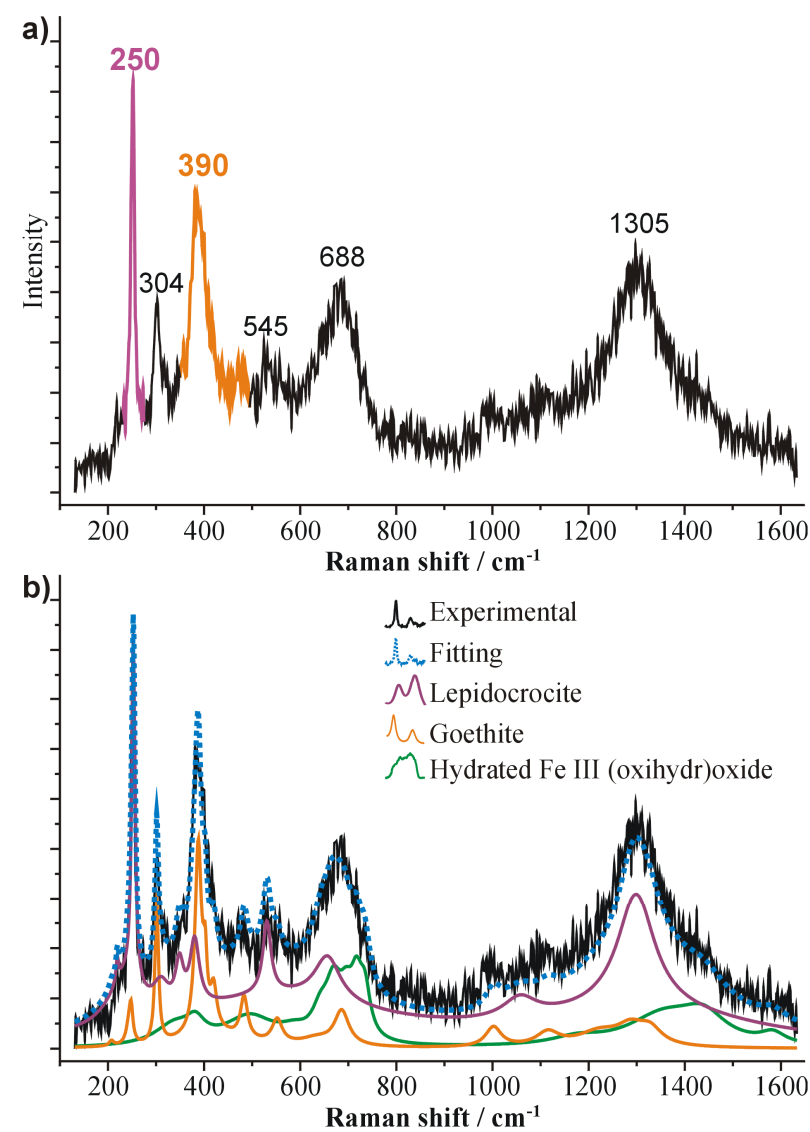

Figure 1. a) Raman spectrum obtained in corrosion products of Am XXX $S$ underlining the phase mixing at the microscopic scale. The $250 \mathrm{~cm}^{-1}$ band is the main band characteristic of lepidocrocite, while the $390 \mathrm{~cm}^{-1}$ is characteristic of goethite. Spectrometer LabRam Infinity, obj. 100x, 300s. b) Decomposition obtained underlining the presence of three components.

approach applied to iron corrosion product quantification was used by Dubois et al. ${ }^{[15]}$. First, experimental spectra are normalised by their intensity. Then each phase spectrum contribution is calculated and the quantification is derived from the calibration curves.

The second approach, already applied at LADIR for organic molecules ${ }^{[16,17]}$ and based on a previous study by Döpner et al. ${ }^{[18]}$, shunts the setting of calibration curves, because assumes that the relative contribution of each compound in the experimental spectrum could be considered as giving directly their quantitative ratio. This assumes that the set of reference compound spectra takes into account each phase with respect to its Raman response intensity. The current available Raman facilities get the required stability to establish "absolute" relative intensities, if the recording of the reference spectra set is realised under the same conditions (for example within the same day). We consider our approach as semi-quantitative because, it needs the previous recognition of compounds that are present and also because of some lack of precision in case of our solid state compounds, as will be discussed latter.

In this work this last approach was chosen because it avoids the establishment of calibration curves which are not always simple to achieve for solids (problems of the homogeneisation of compound mixture for microanalysis). Pre-requirements are therefore limited to the recording of suitable reference compound spectra in the same analytical conditions. 

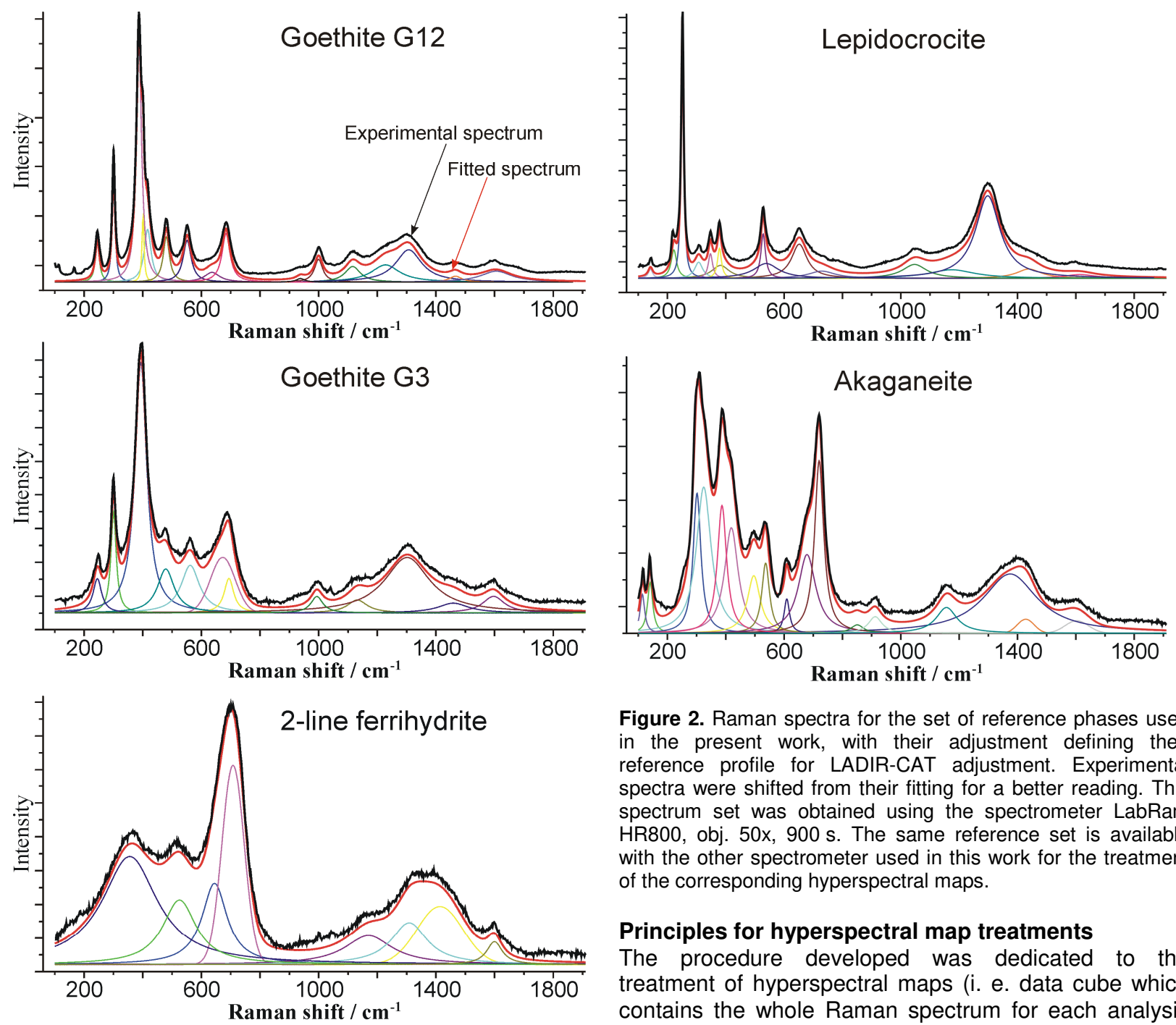

Figure 2. Raman spectra for the set of reference phases used in the present work, with their adjustment defining their reference profile for LADIR-CAT adjustment. Experimental spectra were shifted from their fitting for a better reading. This spectrum set was obtained using the spectrometer LabRam HR800, obj. 50x, $900 \mathrm{~s}$. The same reference set is available with the other spectrometer used in this work for the treatment of the corresponding hyperspectral maps.

\section{Principles for hyperspectral map treatments}

The procedure developed was dedicated to the treatment of hyperspectral maps (i. e. data cube which contains the whole Raman spectrum for each analysis location). For each point analysed the semi-quantitative composition is extracted and, with the data from the area scanned, phase compositional images are constructed. In addition a global estimation of contents for the scanned area is found with the average of all analysed points. Calculations are automated to offer a routine and friendly treatment. For this purpose a homemade application, written in visual basic (programming D. Baron), has been developed: LADIR-CorATmos (LADIR-CAT).

This application includes the mathematical definition of the reference profiles which will be used to fit the experimental spectrum. Because reference spectra should depend on Raman devices and experimental conditions, these reference spectra profiles are established for the sample reference batch acquired for each spectrometer or specific analytical conditions. To avoid possible instrumental drifts, acquisition of the reference set is performed within the same day with a regular control of the setup calibration.

The treatment is then run on spectra sets which compose a hyperspectral map. All spectra are fitted by linear combination of reference profiles to define their quantitative composition. After calculations, a basic graphical interface is available for the visualisation/control of each spectrum and its fit.

The final outputs of the treatment are text files with results calculations (as matrices of numbers or tables) 
a)

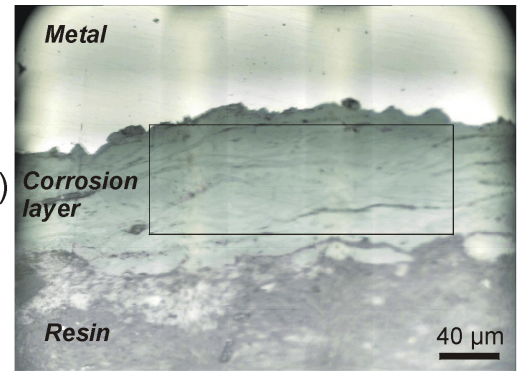

b) Mapping of Goethite contents

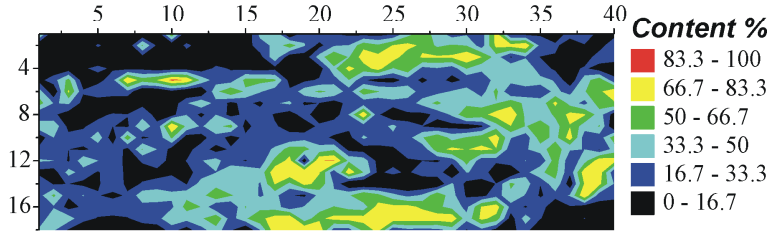

c) Mapping of hydrated Felll (oxihydr)oxide contents

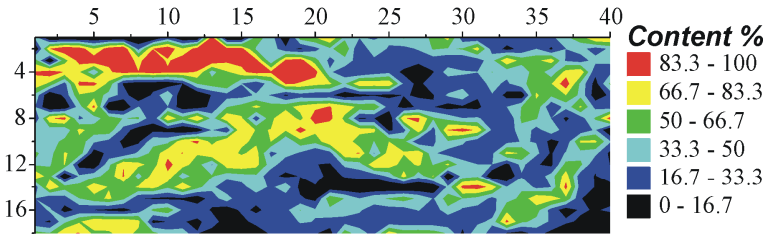

d) Mapping of lepidocrocite contents

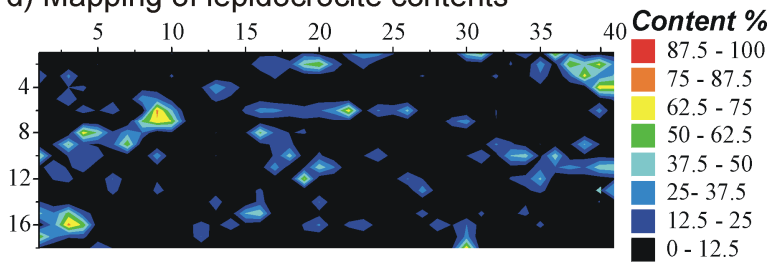

e) Mapping of akaganeite contents

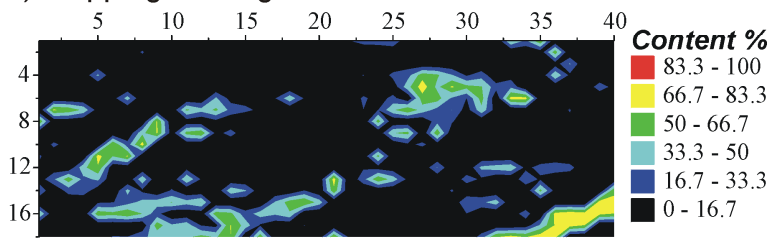

Figure 3. Raman structural imaging of the sample Am XXX S. (a) Optical microphotograph with the location of the scanned area (C5). Semi-quantitative results obtained from spectral decomposition by LADIR-CAT program for (b) goethite (c) hydrated Fe III (oxihydr)oxide. (d) lepidocrocite (e) akaganeite (this figure is available in colour in the online version). Spectrometer LabRam HR800, obj. 50x, mapping $40 \times 18$ spectra (720 points), step $5.5 \times 5.5 \mu^{2}$ (area scanned $214.5 \times 93.5 \mu \mathrm{m}^{2}$ ), acquisition $2 \times 150$ s per spectra. Axes of mapping contents are points of analysis.

which provide two kinds of information about the sample composition. First, matrices for each component (sized by the number of rows and lines in the map), that gives for each cell the compound percent content calculated for the corresponding pixel. This corresponds to compounds contents at each analysed point. Second, a table, giving the average percent content of each component calculated over the whole mapping.

Matrices give images of the sample composition through a 2D contour fill plotting (Fig. 3), where colour contrasts are directly related to quantitative information. The quantitative structural images obtained offer a reliable sample description, unlike the qualitative imaging on
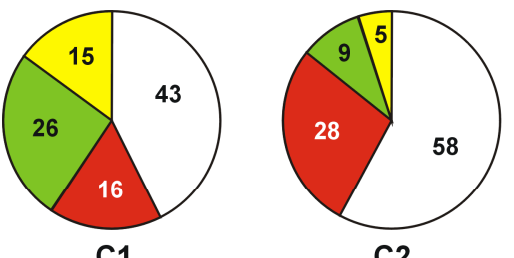

C2
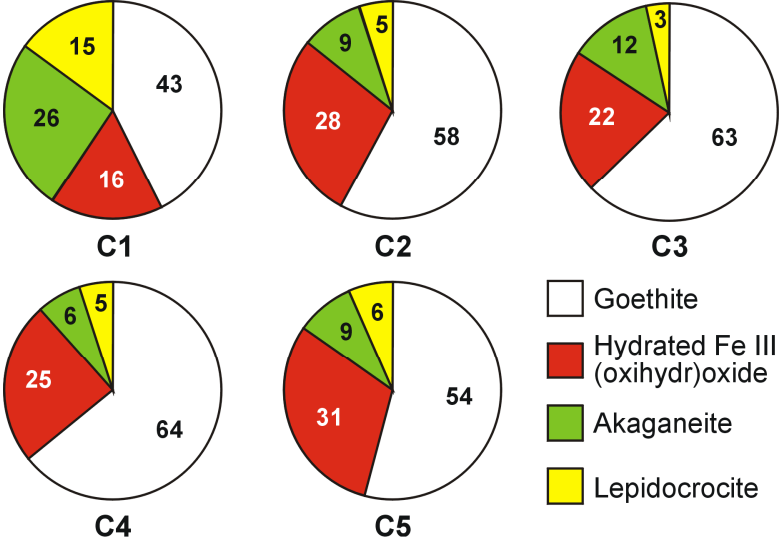

C3

Goethite

Hydrated Fe III (oxihydr)oxide

Akaganeite

Lepidocrocite

Figure 4. Region-based diagrams illustrating the phase content variations for five Raman structural mapping (C1 to C5) on the corrosion product layer of sample Am VI W. Numbers indicate the phase contents in percent. The overall area scanned on this sample is about $1.410^{5} \mu^{2}$.

which colours are user adjusted ${ }^{[1,2,19]}$. However this approach presupposes the recognition of compounds contained in the samples, along with their Raman signatures.

The average percent content over the scanned sample could be illustrated with sector diagrams (Fig. 4) giving "global" sample specificities from microanalysis.

Consistency of the quantitative results obtained could be disturbed by variations of light absorption (Laser and Raman scattering) between pure compounds, mixtures and potential porosities variations in the samples. We hypothesise that these effects have limited consequences (as it will be checked by analysing some prepared mixtures) and lead to errors of the same order than those obtained by the setting of calibration curves $^{[15]}$

\section{Description of LADIR-CAT operations}

Data from hyperspectral map are first prepared to be entered in the program. The hyperspectral object is split in files that correspond to map pixel with names including coordinate of row and line. As dispersive spectrometers provide spectra with non-constant wavenumber interval, a characteristic inappropriate to our calculations, all spectra are loaded in LADIR-CAT environment with resampling at a constant wavenumber step.

An example of a raw set of spectra from a hyperspectral map is shown in Fig. 5. It highlights in a pre-treatment stage the necessity to exclude spikes and fluorescence background. Spikes are mainly a problem for presentation rather than actually a restrictive problem for treatment, but fluorescence contribution must be removed. According to proprietary softwares provided by spectrometers manufacturers, some specific procedures could propose mathematical tools (with sometimes two acquisitions) to overcome these spurious signals. Anyway, the automation of these pre-treatments is not obvious. No ideal solution seems available and best results/control should be obtained by hand treatment.

For LADIR-CAT, to undertake this pre-treatment and to reduce the user intervention we propose some simple automated operations that give acceptable results for the treated data.

Spikes are detected using local slope calculation over each wavenumber step which needs to be lower than a 

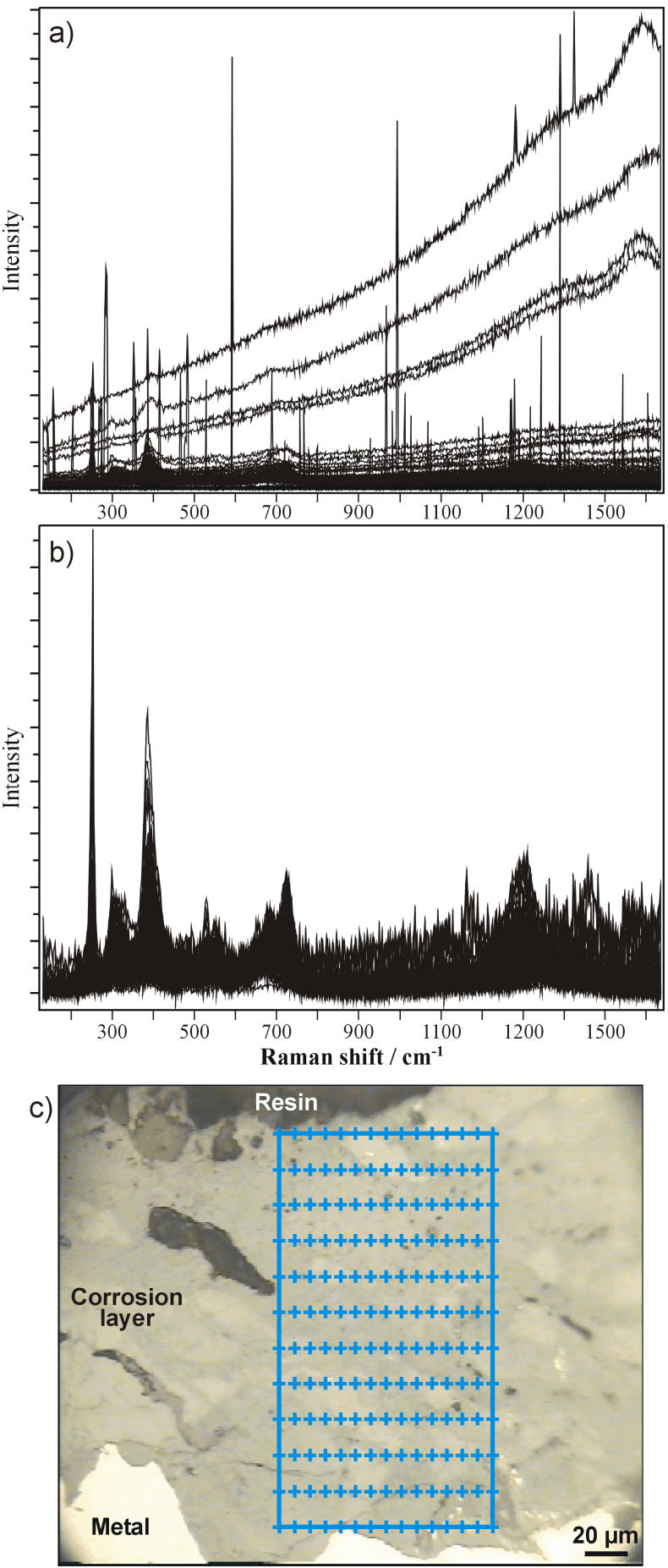

Figure 5. a) Spectra from a hyperspectral map, as acquired without pre-treatment, b) the same data set after baseline correction and spike removal, c) associated micro-photograph. The spectra shown in a) exhibit Raman signatures from iron corrosion phases, fluorescence background mainly from embedding resin, Raman spectra from amorphous carbon remaining from coating for previous SEM analysis (considered here as baseline) and spikes. Sample Ami 01 (C4), spectrometer LabRam Infinity, obj. 50x, mapping 15x12 spectra (180 points), step $7.3 \times 16.4 \mu \mathrm{m}^{2}$, acquisition $2 \times 150$ s per spectra.

user defined threshold. A very strong spectrum increase is therefore interpreted as a spike, and the spectrum is then smoothed whenever it exceeds the tolerated slope. In this study the encountered fluorescent background always presents the same behaviour with a quasi-linear
Measured hyperspectral data set

$$
\downarrow \begin{aligned}
& \text { User or } \\
& \text { automated }
\end{aligned}
$$

Spectra pre-treatment (baselines, spikes)

$$
\downarrow \text { Automated }
$$

Data conditioning: constant wavenumber interval, hyperspectrum splitting

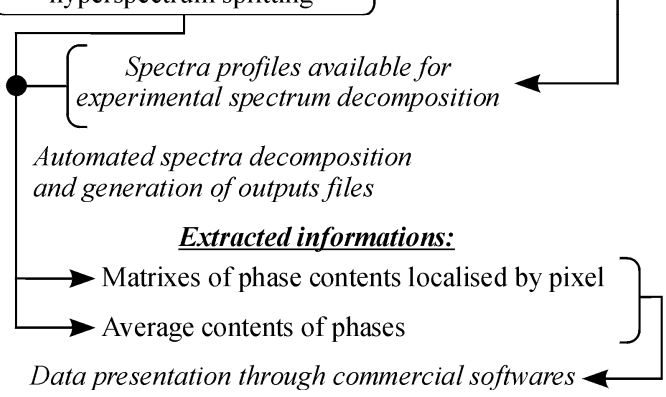

Figure 6. Working scheme of "LADIR-CAT" application.

increasing to high wavenumbers. Therefore a broken line in two segments can simulate the background that has to be removed. These lines are defined by the spectrum extremities and for the inflexion point we chose the intensity at $960 \mathrm{~cm}^{-1}$, close to the middle of the spectrum, and where no significant Raman bands are expected for iron corrosion products.

Two other situations need to be treated in the pretreatment stage. The first one is the detector saturation, arising when fluorescence signal exceeds the CCD detector capacity during the counting time. The second one corresponds to the absence of a significant Raman signal measurement, which occurs when the scanned area overlaps the metallic substrate of the sample. In both situations no meaningful Raman signature can be extracted from these spectra, therefore it was chosen to set them at zero to define a "black pixel" without any identified phase (all settled at zero), which will be excluded from further calculations.

The treatment procedure is summarised in Fig. 6. On the one hand pure phase reference spectra are pre-treated (possible baseline and spike removing), and fitted to provide the set of reference profiles used for decomposition. The LADIR-CAT application defines the reference spectrum profile by a sum of Lorentzian or Gaussian bands on the basis of a pre-existing software module ${ }^{[16,17]}$. The user performs this adjustment with the only constraints of a correct fit of the spectrum using a minimum of bands. In this work these elementary bands will not be used for further interpretation. Fig. 2 presents the set of reference spectra with the adjustment defining their reference profile. On the other hand the Raman maps are pre-treated, adjusted reference spectra profiles are loaded, and decomposition of each spectrum performed.

The experimental spectrum $\left(S p_{\text {exp }}\right)$ is fitted by a linear combination of pure phase spectrum profile $\left(\mathrm{Sp}_{\text {ref }}\right)$ defining the calculated spectrum ( $\left.S p_{\text {calc }}\right)$ :

$$
\mathrm{Sp}_{\text {calc }}=\sum_{\mathrm{i}=1}^{\mathrm{k}} \alpha_{(\mathrm{i})} \mathrm{Sp}_{\text {ref }(\mathrm{i})}
$$

( $\mathrm{k}$ number of reference phase, each i value correspond to one phase) 
The $\alpha_{(k)}$ coefficients are adjusted using a least square fitting procedure undertaken by the LevenbergMarquardt algorithm ${ }^{[20,21]}$ with the minimisation of the quadratic deviation $\mathrm{E}$ :

$$
E=\sum_{j=1}^{n}\left(S p_{\exp (j)}-S p_{\text {calc }(j)}\right)^{2}
$$

(n number of points in the spectrum)

To obtain phase proportions normalised at $100 \%$ (Prop\%(i) ${ }_{(i)}$ in treated spectrum, coefficient for each reference phase is normalised:

$$
\text { Prop } \%_{(\mathrm{i})}=100 \cdot \frac{\alpha_{(\mathrm{i})}}{\sum_{\mathrm{i}=1}^{\mathrm{k}} \alpha_{(\mathrm{i})}}
$$

During experimental spectrum fitting iterations, we do not use criterions to force a positive contribution of a reference spectrum (positive $\alpha_{(\mathrm{k})}$ coefficients). We notice that the attribution at one loop of a negative contribution for one reference phase is associated to the fact that this phase is absent from the analysed point. Therefore we used this criterion to exclude this phase from the reference set (put its content at zero) for this analysed point, and start again the fitting procedure. The fitting iterations are stopped when the mean quadratic deviation between experimental and calculated (adjusted) spectrum is stabilised between two loops in a double precision format (16 significant decimal digits).

\section{Application to iron indoor atmospheric corrosion products: choice of reference spectra}

Previous studies ${ }^{[2,14]}$ have enabled us to define the phase identification and distribution in indoor atmospheric iron corrosion layers as follows: stable goethite constitutes the major corrosion product inside the layer, whereas the active phases lepidocrocite and akaganeite are only locally present in the system, Moreover, other low crystallised phases were identified as active components of the corrosion layer. They are characterised by a weak Raman spectra dominated by a large band around $700 \mathrm{~cm}^{-1}$. Such spectrum could be related to several phases which display similar spectra (Fig. 2): ferrihydrites $\left(\mathrm{Fe}_{5} \mathrm{HO}_{8}, 4 \mathrm{H}_{2} \mathrm{O} ;{ }^{[22]}\right.$ with two types called 2 -line and 6 -line ${ }^{[23]}$ depending on their X-ray diffraction pattern), feroxyhyte $(\delta-\mathrm{FeOOH})^{[24,25]}$ and maghemite in case of a spectrum deteriorated by a poor crystallisation state or a low counting statistic. All these phases are poorly crystallised hydrated iron III oxides or oxihydroxides and at this step their discrimination by their Raman signature is tricky. Complementary measurements by X-ray Absorption Spectroscopy (XAS) and the review of all the Raman spectra obtained on Amiens samples shows that these poorly crystallised phases oscillate between ferrihydrites, feroxyhyte and maghemite poles ${ }^{[14]}$. Therefore in a generic way we will consider such spectrum characterised by a large band around $700 \mathrm{~cm}^{-1}$ as an hydrated Fe III (oxihydr)oxide. Beyond the identified phases, goethite recognised in the studied samples presents different a state of crystallinity underlined by broadened bands of lower intensity (Fig. 7).

The aim of this study was not to determine the structural state of each identified phase but to quantify the composition of the system by phase family defined by their level of reactivity. Thus we chose to retain only the

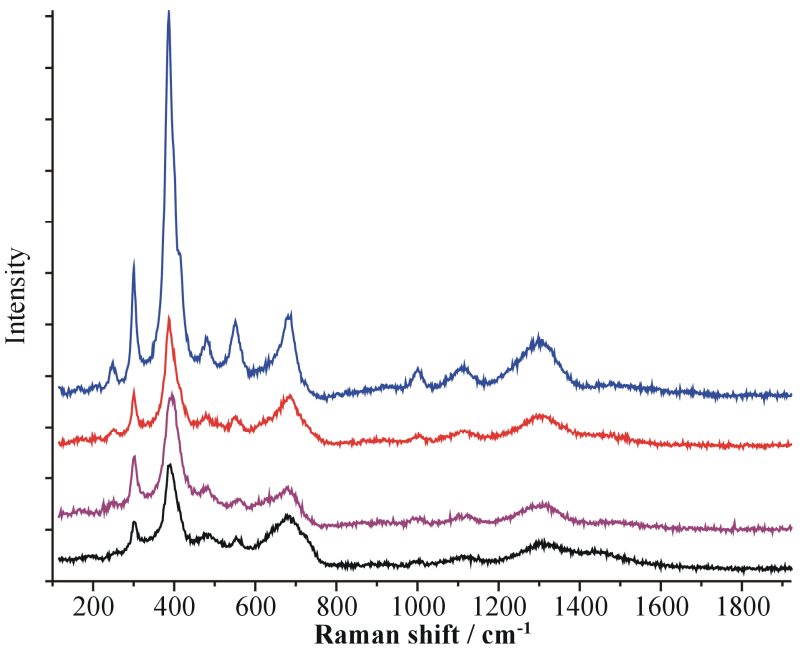

Figure 7. Representative spectra of goethite acquired on sample Am VI W showing its crystallinity state variability. Spectrometer LabRam Infinity, obj. 100x, 150s.

minimum number of reference spectra needed to achieve the proper fitting of recorded spectra. This set defines the "model" chosen to describe the studied system. This is a "model" because although it does not contain a reference spectrum for all the structural state variations encountered, it is sufficient to reproduce experimental spectra.

We noted that for experimental spectra fitting, six phases should be taken into account: goethite, lepidocrocite, akaganeite, 2-line and 6-line ferrihydrite, and maghemite. For a spectrum mainly constituted by a large band around $700 \mathrm{~cm}^{-1}$, at least three phases are kept in the reference set, because experimental spectra shapes could present shape variations between different phases. For goethite, two reference spectra are needed to reproduce the crystallinity variation observed on the analysed samples (Fig. 2).

Seven references therefore constitute the set of reference spectra (Fig. 2), which will define four phase families: lepidocrocite, goethite, akaganeite and an hydrated Fe III (oxihydr)oxide. The sample composition is thus defined by these four components. The amount of goethite is defined by the sum of the identified two reference goethites (called G3 and G12). The hydrated Fe III (oxihydr)oxide component is related to all phases characterised by a weak Raman spectra dominated by a large band around $700 \mathrm{~cm}^{-1}$. Its amount is therefore here described by the sum of ferrihydrites and maghemite spectra identified.

\section{Studied samples: ancient artefacts and reference phases}

Ancient samples studied come from the iron chain bars surrounding the triforium of the Amiens cathedral. These iron chains were placed in 1497 to avoid the collapsing of the nave and transept pillars. The reinforcement is made of 90 bars of about four meters long and $20 \mathrm{~cm}^{2}$ section each, allowing the collection of 31 centimetric samples over the whole structure. Moreover, temperature and relative humidity measurements have shown that the whole cathedral can be considered as a homogeneous environment ${ }^{[26]}$. The precise dating combined with the availability of a great number of samples aged in similar environmental conditions contribute to define the Amiens cathedral as a reference site for the study of indoor atmospheric corrosion. 
Among the collected samples, nine were considered as being enough scanned by Raman mapping to offer a representative analysis, and are presented here (location on Fig. 8).

Concerning the set of reference phases, commercial as well as laboratory synthetic powders were used. Goethite reference powders were synthesised at the LAMBE (UMR 8587), following the protocol proposed by Antony et al. ${ }^{[27,28]}$. Goethite powder labelled G3 was obtained by direct oxidation of metallic iron in $0.1 \mathrm{M}$ $\mathrm{NaOH}$ at near neutral $\mathrm{pH}$ and room temperature. G12 sample was obtained by recrystallisation of ferrihydrite at basic $\mathrm{pH}$ and various temperatures. Lepidocrocite, 2-line and 6-line ferrihydrite references were synthesised following Schwertmann's protocols ${ }^{[22]}$. The synthetic powders were controlled as pure by Infrared spectroscopy, and SEM-EDS analyses confirmed that only $\mathrm{Fe}$ and $\mathrm{O}$ were present. Last, maghemite is a commercial powder from Alfa Aesar as is the additional goethite $(\mathrm{Gc})$ used to prepare validation mixtures.

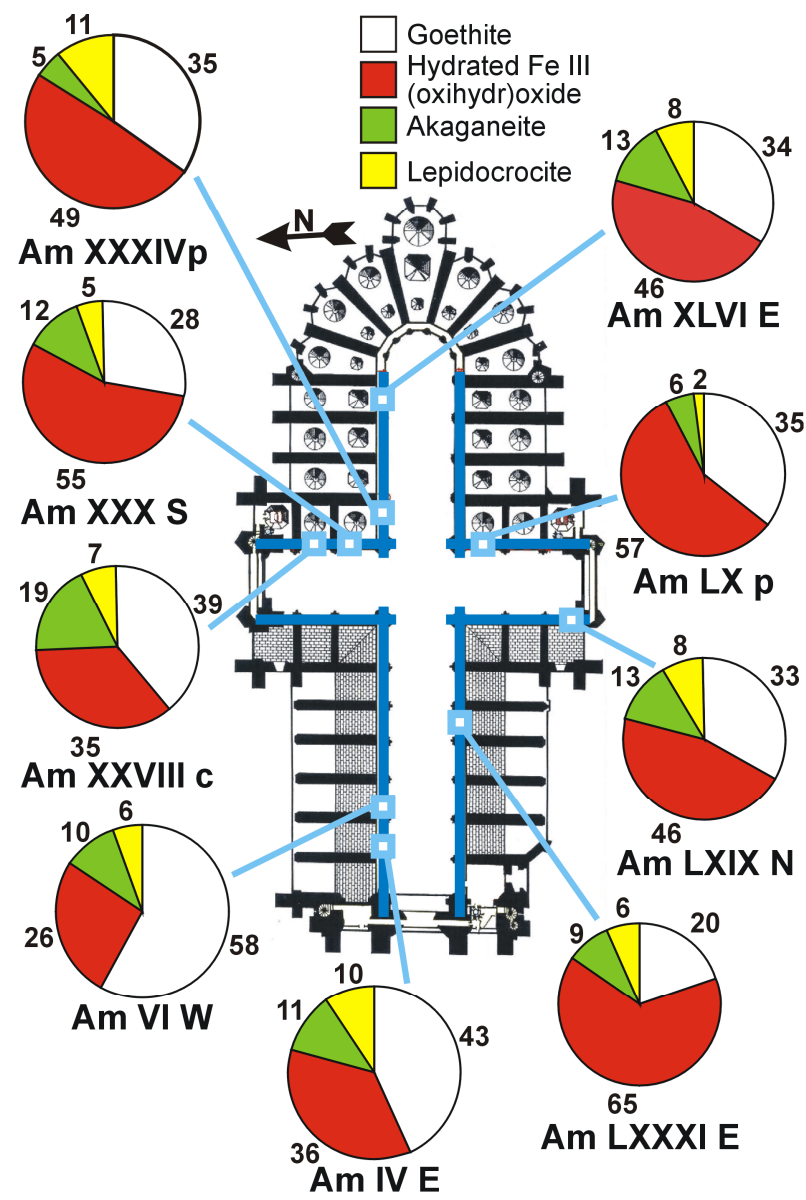

Figure 8. Structural phase contents of the nine samples analysed (values in percent) and their location on the Amiens cathedral plan (see also values in Table 2).

\section{Spectrometers}

Raman spectroscopy measurements were performed at the LADIR using Notch or Edge filter based microspectrometers equipped with Peltier cooled CCD. The first spectrometer used is a LabRam Infinity (Horiba Jobin Yvon) with a focal length of $300 \mathrm{~mm}$ and equipped with a Notch filter, a 1800 lines $/ \mathrm{mm}$ grating and a frequency doubled Nd:YAG Laser emitting at $532 \mathrm{~nm}$. The second one is a LabRam HR800 (Horiba Jobin
Yvon) characterised by a focal length of $800 \mathrm{~mm}$ and Edge filters. For our experiments we use a 600 lines $/ \mathrm{mm}$ grating (allowing the recording in one acquisition of the whole spectral range of our interest) and the $514.5 \mathrm{~nm}$ emission of an air cooled $\mathrm{Ar}^{+}$Laser. Spectral resolution of both set-ups is around $2 \mathrm{~cm}^{-1}$ and calibration is checked on the $520.7 \mathrm{~cm}^{-1}$ band of silicon.

For both devices microanalysis was achieved through Olympus microscopes and objectives. With the objectives used, classic or long working distance, the beams size are about 1 and $1.5 \mu \mathrm{m}$ for a $100 \mathrm{x}$, or about 2.5 and $4 \mu \mathrm{m}$ for a $50 x$ respectively.

These high luminosity devices allow the recording of Raman spectra even with the very low Laser excitation power which is needed to avoid thermal modification of iron oxides or oxi-hydroxides ${ }^{[29]}$. With our spectrometer configurations we have checked that under a 100x objective, a Laser power at sample (modulated by density filters) of about $100 \mu \mathrm{W}$ (or about $120 \mu \mathrm{W}$ for a $50 x$ objective) allows to avoid the thermal modification of all analysed iron corrosion products. Spectra acquisitions were managed by the LabSpec software (Horiba Jobin Yvon), which also drives the recording of spectral maps by controlling an automated $X Y$ microscope stage.

\section{Results and discussion}

Validation of the proposed quantitative approach

A cross-validation of quantitative results through other structural characterisation methods is difficult for our corroded samples. With X-ray diffraction (XRD), that could be performed with a micro-beam on the corrosion layers, an adjusted quantification by Rietveld refinement will fail because of the large presence of low crystallised phases exhibiting broad diffraction patterns ${ }^{[30]}$. With Mössbauer spectroscopy, the sampling of a representative amount of powder is difficult because of the extremely high adherence of the corrosion products on the metal surface. Moreover the "global" analysis by Mössbauer spectroscopy will need some hypothesis from other methods on the system composition to provide univocal solutions.

Therefore as a first approach, the validation of quantitative structural compositions was tested on different mixtures of synthetic powders. A set of four mass ratio of sintered powder pellets of lepidocrocite/goethite mixtures was prepared. For each pellet six measurements using a 50x objective were performed, in order to take into account the possible heterogeneity of the mixtures and reproducibility of the procedure.

Results between the expected and the measured compositions are given in Table 1 and illustrated in Fig. 9. The relative difference between the mean measured contents for the mixtures and the theoretical ones, are within $8 \%$. The dispersion of the measurements for one sample (standard deviation below $7 \%$ ) is interpreted as related to a rough homogeneity of the analysed mixtures, more than to a low reliability of the quantification procedure. Even if lower phase contents seem underestimated in these experiments (inducing an overestimation of the major phase) the quantification shows a quite fair accuracy, suitable for our application of phase content estimation in corrosion layers (semi-quantitative approach). 


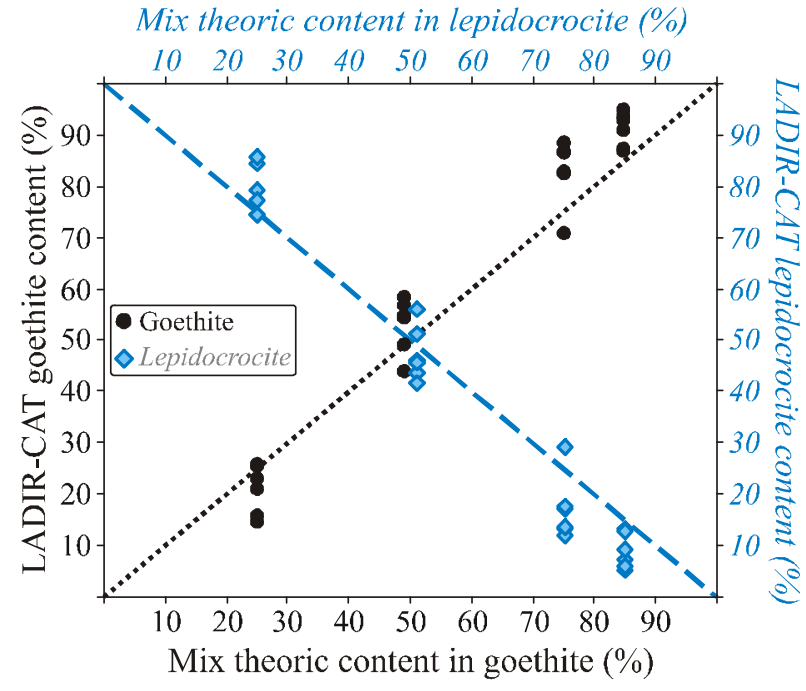

Figure 9. Plot of the contents obtained by LADIR-CAT procedure versus the theoretical content for several mixing proportion of goethite (black) and lepidocrocite (blue) (see also Table 1). Dotted lines represent the exact correspondence between calculated and expected contents. Symbols for the $50 / 50$ sample are slightly horizontally shifted for a better reading.

\begin{tabular}{|c|c|c|c|c|}
\hline $\begin{array}{c}\text { Mixing theoric } \\
\text { content } \\
\text { G: goethite, } \\
\text { L: lepidocrocite }\end{array}$ & \multicolumn{2}{|c|}{$\begin{array}{c}\text { LADIR-CAT calculated } \\
\text { goethite content }\end{array}$} & \multicolumn{2}{|c|}{$\begin{array}{c}\text { LADIR-CAT calculated } \\
\text { lepidocrocite content }\end{array}$} \\
\cline { 2 - 5 } & $\langle\mathrm{m}>(\%)$ & $\sigma(\%)$ & $\langle\mathrm{m}>(\%)$ & $\sigma(\%)$ \\
\hline G 25 \% - L 75 \% & 21 & 5 & 79 & 5 \\
\hline G 50\% - L 50\% & 53 & 6 & 47 & 6 \\
\hline G 75 \% - L 25\% & 83 & 7 & 17 & 7 \\
\hline G 85\% - L 15\% & 91 & 4 & 9 & 4 \\
\hline
\end{tabular}

$<\mathrm{m}>$ : mean and $\sigma$ : standard deviation

Table 1. Results (mean and standard deviation for six measurements) of the contents determination obtained by LADIR-CAT procedure on five mixtures of goethite and lepidocrocite reference powders (see also Fig. 9).

To extend this validation, some mixtures of goethite and 2-line ferrihydrite were also prepared, but even with a careful preparation the obtained pellets are highly heterogeneous. The quantification results obtained by LADIR-CAT on these mixtures are too scattered to assess the quantification procedure. This problem underlines the limits of the validation by mixing of standard reference powders, because mixture homogenisation is sometimes difficult to realise.

\section{Quantification of Amiens' cathedral corrosion products}

Our previous studies ${ }^{[2,14]}$ and the Raman characterisation of the present samples (points analysis, mapping and qualitative phase distribution) show that the corrosion systems developed on very long term are very heterogeneous. However, by scanning at least ten percent of the sample surface at various places through several Raman mapping, one can assume to achieve quite a representative view of the sample. We chose to perform mapping with a $50 x$ magnification, and a square step size between $5 \times 5$ and $8 \times 8 \mu m^{2}$ in order to reasonably probe the scanned area for each map. Spectrum acquisition takes about 300 seconds to get a good signal/noise ratio and thus a reliable decomposition. As a consequence, the acquisition of the hyperspectral maps performed was between 15 hours (for 180 points) to 85 hours (for 1020 points).

All the acquired maps were treated using LADIR-CAT. During the treatments, the suitability of the reference phase set was checked, by the control of the accuracy of the obtained fitting. It proved to describe correctly the phases mixtures present in the samples.

Figure 3 presents an example of quantitative images of the phases with their locations and their respective ratio. In a conservative approach we chose to present images from 2D contour maps with a limited number of colour levels. Here six colours levels were fixed by content step of $16.7 \%$. Only Lepidocrocite was presented using eight colour levels (steps of $12.5 \%$ ). Because of the Lepidocrocite intense band at $250 \mathrm{~cm}^{-1}$, we are confident in its right detection even at low content. Even if the treatment provides quantitative contents, we prefer such representation by compositional steps in order to avoid any over-interpretation, without expecting a precision which could not be guaranteed.

Using the obtained images, a detailed structural mapping of the corrosion layer was achieved. Phase distribution in the corrosion layer is as follows: the main matrix is composed of Goethite with other phases locally present. Lepidocrocite is located more generally in the external part of the corrosion layer, often (but not only) correlated with the presence of cracks which are sometimes in the vicinity of the metallic substrate. Akaganeite is mostly located along cracks in the external part of the corrosion layer. The rest of the analysed area is constituted of hydrated iron III (oxihydr)oxide mainly organised as marblings ${ }^{[13]}$. These marblings, sometimes connected to the metallic substrate, are highly susceptible to play an active role in the corrosion processes $^{[14]}$.

In addition to the $2 \mathrm{D}$ images of contents that informs about the phase organisation and the related corrosion mechanisms, we have also estimated the global phase content in the analysed volume. To take into account the heterogeneity of the corrosion layers, it was necessary to obtain several hyperspectral maps for each samples. Figure 4 illustrates this and presents the phase proportions, as region-based diagrams, corresponding to the five Raman mapping registered on sample Am VI W. The lepidocrocite and akaganeite contents in the global composition of $\mathrm{C} 1$ mapping are three to five times higher than in the other mapping, and therefore the goethite and the hydrated iron III (oxihydr)oxide phase contents are consequently lower. This variation is taken into account by calculating for each sample the "global region-based diagram". This diagram is calculated on the basis of an average, weighted by scanned areas of the maps acquired on one sample, in order to avoid an influence of a "particular" area on a sample. Table 2 details the compositions obtained for the nine samples collected around the triforium, giving for each samples: the total number of point analysed, the mean phase content, the weighted mean, the standard deviation, the minimum and maximum. The deduced global regionbased diagrams are presented in Fig. 8. 


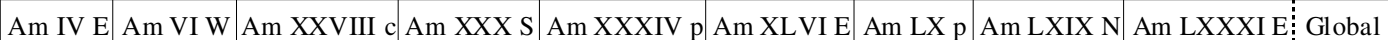

\begin{tabular}{|c|c|c|c|c|c|c|c|c|c|c|c|}
\hline \multicolumn{2}{|c|}{$\begin{array}{l}\text { Total number of } \\
\text { points analysed }\end{array}$} & 4913 & 2447 & 3391 & 2043 & 1144 & 3108 & 2059 & 2422 & 2465 & 23992 \\
\hline \multirow{4}{*}{ 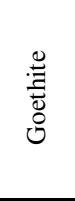 } & $<\mathrm{m}>(\%)$ & 43 & 58 & 39 & 28 & 35 & 34 & 35 & 33 & 20 & 36 \\
\hline & $\sigma(\%)$ & 15 & 8 & 4 & 6 & 9 & 12 & 33 & 11 & 9 & 11 \\
\hline & $\min (\%)$ & 15 & 43 & 32 & 22 & 28 & 20 & 4 & 25 & 14 & 4 \\
\hline & $\max (\%)$ & 56 & 64 & 43 & 37 & 47 & 53 & 72 & 56 & 38 & 72 \\
\hline \multirow{4}{*}{ 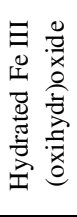 } & $<\mathrm{m}>(\%)$ & 36 & 26 & 35 & 55 & 49 & 46 & 57 & 46 & 65 & 46 \\
\hline & $\sigma(\%)$ & 13 & 6 & 13 & 9 & 7 & 10 & 31 & 7 & 6 & 11 \\
\hline & $\min (\%)$ & 21 & 17 & 23 & 42 & 45 & 38 & 24 & 37 & 54 & 17 \\
\hline & $\max (\%)$ & 69 & 31 & 57 & 64 & 60 & 63 & 88 & 57 & 70 & 88 \\
\hline \multirow{4}{*}{ 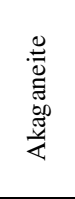 } & $<\mathrm{m}>(\%)$ & 11 & 10 & 19 & 12 & 4 & 13 & 6 & 13 & 9 & 11 \\
\hline & $\sigma(\%)$ & 7 & 8 & 7 & 5 & 1 & 8 & 3 & 6 & 3 & 5 \\
\hline & $\min (\%)$ & 2 & 6 & 10 & 6 & 4 & 1 & 2 & 1 & 5 & 1 \\
\hline & $\max (\%)$ & 17 & 26 & 29 & 19 & 6 & 19 & 8 & 17 & 13 & 29 \\
\hline \multirow{4}{*}{ 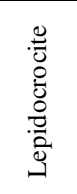 } & $<\mathrm{m}>(\%)$ & 10 & 6 & 7 & 5 & 11 & 8 & 2 & 8 & 6 & 7 \\
\hline & $\sigma(\%)$ & 8 & 5 & 5 & 2 & 8 & 7 & 2 & 4 & 2 & 5 \\
\hline & $\min (\%)$ & 0 & 3 & 0 & 2 & 1 & 3 & 0 & 4 & 3 & 0 \\
\hline & $\max (\%)$ & 14 & 15 & 14 & 7 & 21 & 22 & 6 & 15 & 10 & 22 \\
\hline
\end{tabular}

$<\mathrm{m}>$ : weighted mean by analysed surface ; $\cong$ : standard deviation ; min and max: minimum and maximum contents measured among the maps realised. For global values are indicated: the mean of means values, the standard deviation of means values, the overall minimum and maximum.

Table 2. Results of the contents determination obtained by LADIR-CAT procedure on nine Amiens samples (see also Fig. 8).

Goethite and hydrated iron III (oxihydr)oxide phases are the major phases. Lepidocrocite and Akaganeite are present in much lower concentration, always below $20 \%$. Considering the content standard deviation for each phase (table 2), we can conclude that the phase proportions are globally the same all around the triforium: $40 \%$ Goethite, $40 \%$ hydrated iron III (oxihydr)oxide, $10 \% \quad$ Lepidocrocite and $10 \%$ Akaganeite. These results enlighten the great amount of low crystallised phases in the corrosion layers developed on Amiens' chains. These phases should therefore be integrated when considering the corrosion scale reactivity, and their proportions have to be integrated in the "protective ability ratio" used to define the corrosion product layer reactivity. The LADIR-CAT program is therefore likely to be a way for supplying data for the corrosion diagnosis, through the calculation of the ratio between the stable phases and the reactive ones.

\section{Conclusion}

The LADIR-CAT program presents a specific approach to extract quantitative information from hyperspectral Raman maps. It is based on the spectral decomposition of experimental spectra by a linear combination of reference spectra. The important point in the process is to take into account the respective Raman intensity response, by the acquisition of a suitable reference spectra set. A first validation was performed through the analysis of synthetic powders mixtures. It had underlined quite a fair accuracy between calculated and expected compositions. This procedure is therefore assumed to provide reliable quantitative results for the studied systems. Obviously a cross-validation implemented on ancient samples through an other independent technique, which remains to be defined, would be needful.

The program was especially developed for treatment of hyperspectral maps, rather than the quantitative calculations for one spectrum, and it therefore offers an easy interface for this purpose. This application has been tested in the context of atmospheric corrosion on corroded layers from the iron chains of the Amiens cathedral. The application to corrosion product layers shows the possibility to draw 2D-images describing quantitatively the spatial organisation of the oxidised phases. As a consequence, this first application is wellsuited to the complex heterogeneous systems encountered in the corrosion field. Moreover, the quantitative results obtained enable to better understand the corrosion product layer reactivity. In particular, the proportion of high reactive phases like hydrated iron III (oxihydr)oxide has been quantified.

These results will help defining a new protective ability ratio that should improve the diagnosis method of iron degradation in the context of cultural heritage artefacts. It will also permit to improve the modelling of atmospheric corrosion mechanisms for the nuclear waste disposal.

\section{Acknowledgments}

This study was supported by the PNRC project "Mise en place d'une méthode de diagnostic de la corrosion atmosphérique" of the Ministère de la Culture et de la Communication, by the GdR 3174 of CNRS "ChimARC", 
and the ARCOR project supported by the French National Research Agency (ANR). We kindly thank Ludovic Legrand (LAMBE) for the preparation of reference samples, and we friendly thank Céline Paris (LADIR) for her precious help in the micro-Raman mapping acquisitions.

\section{References}

[1] L. Bellot-Gurlet, D. Neff, S. Réguer, J. Monnier, M. Saheb, P. Dillmann, Journal of Nano Research 2009, 8, 147.

[2] D. Neff, L. Bellot-Gurlet, P. Dillmann, S. Reguer, L. Legrand, Journal of Raman Spectroscopy 2006, 37, 1228.

[3] S. Réguer, D. Neff, L. Bellot-Gurlet, P. Dillmann, Journal of Raman Spectroscopy 2007, 38, 389.

[4] L. S. Selwyn, P. J. Sirois, V. Argyropoulos, Studies in Conservation 1999, 44, 217.

[5] H. Antony, S. Perrin, P. Dillmann, L. Legrand, A. Chaussé, Electrochimica Acta 2007, 52, 7754.

[6] U. R. Evans, C. A. J. Taylor, Corrosion Science $1972,12,227$.

[7] M. Stratmann, K. Bohnenkamp, H.-J. Engell, Werkstoffe und Korrosion 1983, 34, 604.

[8] M. Yamashita, H. Miyuki, Y. Matsuda, H. Nagano, T. Misawa, Corrosion Science 1994, 36, 283.

[9] S. Hoerlé, F. Mazaudier, P. Dillmann, G. Santarini, Corrosion Science 2004, 46, 1431.

[10] K. Kashima, S. Hara, H. Kishikawa, H. Miyuki, Corrosion Engineering 2000, 49, 25.

[11] H. Kihira, T. Misawa, T. Kusunoki, K. Tanabe, T. Saito, Corrosion Engineering 1999, 48, 979.

[12] J. Monnier, PhD Thesis, Université Paris-Est, available on line at http://tel.archives-ouvertes.fr/tel00369510/fr/ 2008

[13] J. Monnier, L. Legrand, L. Bellot-Gurlet, E. Foy, S. Reguer, E. Rocca, P. Dillmann, D. Neff, F. Mirambet, S. Perrin, I. Guillot, Journal of Nuclear Materials 2008, 379, 105

[14] J. Monnier, D. Neff, S. Réguer, P. Dillmann, L. Bellot-Gurlet, E. Leroy, E. Foy, L. Legrand, I. Guillot, Corrosion Science 2010, 52, 695.

[15] F. Dubois, C. Mendibide, T. Pagnier, F. Perrard, C. Duret, Corrosion Science 2008, 50, 3401.

[16] S. Bernad, T. Soulimane, S. Lecomte, Journal of Raman Spectroscopy 2004, 35, 47.

[17] F. Salpin, F. Trivier, S. Lecomte, C. Coupry, Journal of Raman Spectroscopy 2006, 37, 1403.

[18] S. Döpner, P. Hildebrandt, G. A. Grant Mauk, H. Lenk, W. Stempfle, Spectrochimica Acta Part A: Molecular and Biomolecular Spectroscopy 1996. 52, 573.

[19] G. Gouadec, L. Bellot-Gurlet, D. Baron, P. Colomban, in Raman imaging, Springer-Verlag, 2010.

[20] K. Levenberg, The Quarterly of Applied Mathematics 1944, 2, 164.

[21] D. Marquardt, SIAM Journal on Applied Mathematics 1963, 11, 431.

[22] U. Schwertmann, J. Friedl, A. Kyek, Clays and Clay Minerals 2004, 52, 221.

[23] L. Mazzetti, P. J. Thistlethwaite, Journal of Raman Spectroscopy 2002, 33, 104.

[24] N. Boucherit, P. Delichere, S. Joiret, A. Hugot-Le Goff, Material Science Forum 1989, 44-45, 51.
[25] G. Nauer, P. Strecha, N. Brinda-Konopik, G. Liptay, Journal of Thermal Analysis 1985, 30, 813.

[26] J. Monnier, L. Bellot-Gurlet, L. Legrand, P. Dillmann, S. Réguer, D. Neff, I. Guillot, in Metal07, Vol. 2 (Eds.: C. Degrigny, R. v. Langh, I. Joosten, B. Ankersmit), Published by ICOM-CC, Amsterdam, 2007, pp. 47.

[27] H. Antony, Thesis thesis, Université d'Evry (Evry), 2005.

[28] V. Lair, H. Antony, L. Legrand, A. Chausse, Corrosion Science 2006, 48, 2050.

[29] D. Neff, S. Reguer, L. Bellot-Gurlet, P. Dillmann, R. Bertholon, Journal of Raman Spectroscopy 2004, 35, 739.

[30] P. Keller, Werstoffe und Korrosion 1967, 18, 865. 